\title{
ON THE INTERLACING PROPERTY OF STABLE COMPLEX SYSTEMS OF DIFFERENTIAL EQUATIONS
}

\author{
ZIAD ZAHREDDINE
}

\begin{abstract}
By exploiting recent stability results, an interesting property known in stability theory as the interlacing property is revisited and reproduced. The approach is straightforward and highlights the central role that positive para-odd functions is currently playing in the stability of complex systems of differential equations.
\end{abstract}

\section{Introduction}

The interlacing property plays a crucial role in the stability theory of differential equations. Using this property, Kharitonov [2] and [3] obtained the well-known Kharitonov theorems for real interval polynomials and recently, Argoun [1] applied it to the stability of perturbed systems in the real case. For more applications of the interlacing property in the stability of perturbed real systems, see for example [4] and [5] where in the latter the terminology Hermite-Biehler theorem was used instead of interlacing property.

The technique we shall use applies to both real and complex systems. We intend to reproduce the interlacing property by resorting to the theory of positive para-odd functions which is playing an increasing effective role in the stability of differential equations with complex coefficients [6]. The main focus of this paper is the straightforwardness with which such a property is obtained without necessarily evoking Tellegen's theorem or the complex counterpart of Foster's reactance theorem. Our approach applies to both continuous and discrete-time systems showing again [7] the effectiveness of positive para-odd functions in displaying the intimate connections between Routh-Hurwitz and Schur-Cohn types of stability [8].

Received May 25, 1993.

1991 Mathematics Subject Classification. Primary 93D, Secondary 34D.

Key words and phrases. Interlacing property, Hermite Biehler Theorem, positive para-odd functions. 
In section 2, we exploit to advantage the relationship between a stable system and a positive para-odd function and we settle the interlacing property for continuous-time systems. The discrete-time counterpart is dealt with in section 3 .

\section{Continuous-Time Systems}

Consider a continuous time system (real or complex) with characteristic polynomial given by

$$
f(z)=z^{n}+a_{1} z^{n-1}+\ldots+a_{n-2} z^{2}+a_{n-1} z+a_{n} .
$$

Define

$$
h(z)= \begin{cases}\frac{f-f^{*}}{f+f^{*}} & \text { if } n \text { odd } \\ \frac{f+f^{*}}{f-f^{*}} & \text { if } n \text { even, }\end{cases}
$$

where $f^{*}$ the paraconjugate of $f$ is defined by

$$
f^{*}(z)=\overline{f(-\bar{z})}=(-1)^{n} z^{n}+(-1)^{n-1} \bar{a}_{1} z^{n-1}+\ldots+\bar{a}_{n-2} z^{2}-\bar{a}_{n-1} z+\bar{a}_{n} .
$$

Then $h$ takes the form

$$
h(z)=\frac{z^{n}+i \operatorname{Im} a_{1} z^{n-1}+\operatorname{Re} a_{2} z^{n-2}+i \operatorname{Im} a_{3} z^{n-3}+\operatorname{Re} a_{4} z^{n-4}+\ldots}{\operatorname{Re} a_{1} z^{n-1}+i \operatorname{Im} a_{2} z^{n-2}+\operatorname{Re} a_{3} z^{n-3}+i \operatorname{Im} a_{4} z^{n-4}+\ldots} .
$$

It is clear from [6], that if (1) is stable (all zeros of $f$ in Re $z<0$ ), then $h$ may be written in the partial fraction expansion form

$$
h(z)=a_{0}+d_{0} z+\frac{d_{1}}{z-i w_{1}}+\frac{d_{2}}{z-i w_{2}}+\ldots+\frac{d_{n-1}}{z-i w_{n-1}}
$$

where $\operatorname{Re} a_{0}=0, d_{j} \geq 0$ for $j=0,1, \ldots, n-1$ and where the $w_{j}$ are distinct real numbers. Any function satisfying the above expansion is called positive para-odd [6].

We recall the following

Theorem 1. ([6], Theorem 3.1)

(1) is stable if and only if the partial fraction expansion of $h$ satisfies $d_{j}>0$ for $j=0,1, \ldots, n-1$.

The following theorem shows how the interlacing property can be used as a stability test for systems of differential equations.

Theorem 2. (Interlacing Property)

The system (1) is stable if and only if Re $a_{1}>0$ and the zeros and poles of $h$ are simple, lie on the imaginary axis and interlace.

Proof. If (1) is stable, then $h$ can be written as in Theorem 1. From the analysis done on $h$ in [6], $a_{0}+d_{0} z$ represents the quotient obtained when the numerator of $h$ is 
divided by its denominator, hence $d_{0}=\frac{1}{\text { Rea }}$ leading to Re $a_{1}>0$. From the partial fraction expansion of $h$, it follows that the poles of $h$ are simple and imaginary and the same applies on the roots, for it is clear that $h$ is positive para-odd if and only if $\frac{1}{h}$ is positive para-odd.

Without loss of generality, we assume that $a_{0}=0$. (Otherwise we take $H=h-a_{0}$, and we do our analysis on $H$ ).

Let $k(s)=-i \hbar(i s)$, then

$$
\begin{aligned}
& k(s)=d_{0} s-\frac{d_{1}}{s-w_{1}}-\frac{d_{2}}{s-w_{2}}-\ldots-\frac{d_{n-1}}{s-w_{n-1}}, \text { and } \\
& k^{\prime}(s)=d_{0}+\frac{d_{1}}{\left(s-w_{1}\right)^{2}}+\frac{d_{2}}{\left(s-w_{2}\right)^{2}}+\ldots+\frac{d_{n-1}}{\left(s-w_{n-1}\right)^{2}} .
\end{aligned}
$$

Therefore $k^{\prime}(s)>0$ for $s \neq w_{k}$,

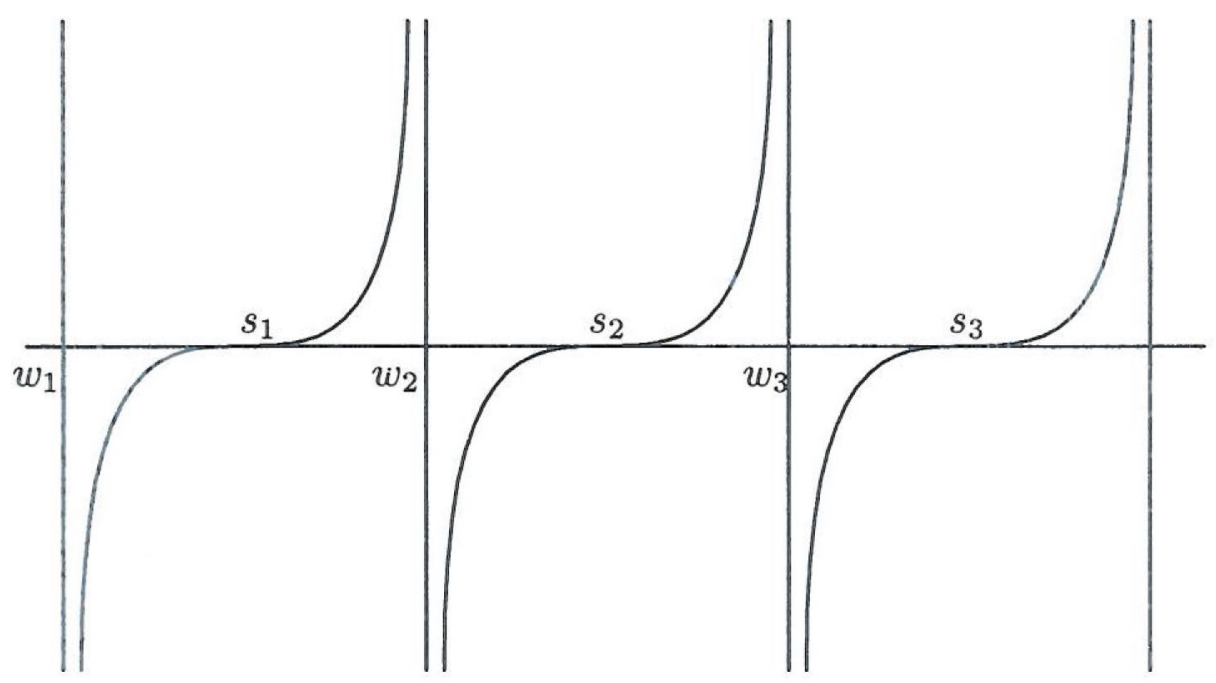

and $k(s)$ is strictly increasing from $-\infty$ to $\infty$ in each interval $\left(w_{j}, w_{j+1}\right)$ and the equation $k(s)=0$ has exactly one solution on each of these intervals, leading to the desired conclusion.

Conversely, let $i s_{1}, i s_{2}, \ldots, i s_{n}$ and $i w_{1}, \ldots, i w_{n}$ be respectively the roots and poles of $h$ such that

$$
s_{1}<w_{1}<s_{2}<w_{2}<\ldots<s_{n-1}<w_{n-1}<s_{n} .
$$

Let

$$
h(z)=a_{0}+d_{0} z+\frac{d_{1}}{z-i w_{1}}+\frac{d_{2}}{z-i w_{2}}+\ldots+\frac{d_{n-1}}{z-i w_{n-1}}
$$

be the partial fraction expansion of $h$. Again $d_{0}=\frac{1}{R e a_{1}}$ and $d_{0}>0$. Also, we may supose $a_{0}=0$. Reconsider the form

$$
k(s)=-i h(i s)=d_{0} s-\frac{d_{1}}{s-w_{1}}-\frac{d_{2}}{s-w_{2}}-\ldots-\frac{d_{n-1}}{s-w_{n-1}} .
$$


From (2), it follows automatically that $d_{j}>0$ for $j=1, \ldots, n$ and by ([6], Theorem 3.1), (1) is stable.

\section{Discrete-Time Systems}

The ideas and results of section 2 may be conveyed to discrete systems in the following way:

Let

$$
g(w)=w^{n}+a_{1} w^{n-1}+\ldots+a_{n}
$$

be the characteristic polynomial of a discrete system (real or complex). Define

$$
g^{T}(w)=w^{n} f\left(\frac{1}{w}\right)=a_{n} w^{n}+a_{n-1} w^{n-1}+\ldots+1
$$

The discrete-time counterpart of Theorem 2 is:

Theorem 3. The system (3) is stable (all roots of $g$ inside the unit circle) if and only if $\left|\frac{a_{n}}{a_{1}}\right|<1$ and $g-g^{T}$ and $g+g^{T}$ have interlacing roots on the unit circle.

Using the bilinear transformation $w=\frac{z-1}{z+1}$ (or $z=\frac{1+w}{1-w}$ ), the proof of Theorem 3 may be traced in the spirit of [7] and [8] where explicit relationships between RouthHurwitz (continuous systems) and Schur-Cohn (discrete systems) types of stability have been established.

\section{References}

[1] M.B. Argoun, "Frequency domain conditions for the stability of perturbed polynomials," IEEE Trans. Aut. Cont, vol. AC-32, PP.913-916, 1987.

[2] V.L. Kharitonov, "Asymptotic stability of an equilibrium position of a family of systems of linear differential equations," Differential Uravnen, vol. 14, no 11, PP.2086-2088, 1978.

[3] V.L. Kharitonov, "On a generalization of a stability criterion," Izv. Akad. Navk. Kazakh. SSR Ser. Fiz. Mat. (in Russian), vol. 1, PP.53-57, 1978.

[4] C.B. Soh, "Damping margin of continuous-time systems with polytope uncertainties," Int. J. systems. Sci., vol. 21, no 4, PP. 749-754, 1990.

[5] Y.C. Soh, "Strict Hurwitz property of polynomials under coefficient perturbation," IEEE Trans. Aut. Cont., vol 34, no 6, PP.629-632, 1989.

[6] Z. Zahreddine, "An Extension of the Routh Array for the Asymptotic stability of a System of Differential Equations With Complex Coefficients," Applicable Analysis, vol. 49, PP. 61-72, 1993.

[7] Z. Zahreddine, "A Routh-Hurwitz Approach to A Schur-Cohn Problem," Indian Journal of Pure and Applied Mathematics, to appear.

[8] Z. Zahreddine, "Explicit Relationships Between Routh-Hurwitz And Schur-Cohn Types of stability," Bulletin of The Irish Mathematical society, to appear.

Department of Mathematics, U.A.E. University, Al-Ain. P.O. Box 17551, United Arab Emirates. 